\section{Pré-diabetes: diagnóstico, avaliação de complicações crônicas e tratamento}

\author{
Prediabetes: diagnosis, evaluation of chronic \\ complications, and treatment
}

Camila Furtado de Souza ${ }^{1,2}$, Jorge Luiz Gross², Fernando

Gerchman², Cristiane Bauermann Leitão²

\section{SUMÁRIO}

O diabetes melito do tipo 2 (DM2) é responsável por $90 \%$ dos casos de diabetes e está associado a complicações micro e macrovasculares de elevada morbimortalidade. Os indivíduos com maior risco de desenvolvimento de DM incluem aqueles com glicemia de jejum alterada (GJA) e tolerância diminuída à glicose (TDG) e especialmente aqueles com as duas condições combinadas. Esses indivíduos fazem parte de um grupo hoje conhecido como pré-diabetes. Aproximadamente $25 \%$ dos indivíduos com pré-diabetes desenvolverão DM2 em três a cinco anos. A hiperglicemia, na ausência de DM, também foi associada ao aumento no risco de doença cardiovascular. Estudos demonstraram que mudanças no estilo de vida e intervenções medicamentosas são efetivas em retardar ou prevenir o DM2 em pacientes com pré-diabetes. GJA e TDG estão associadas ao desenvolvimento de DM2 e, apesar das controvérsias, a maioria dos estudos epidemiológicos reforça a importância dessas duas condições também no desenvolvimento de doença micro e macrovascular. Assim, intervenções em pacientes com pré-diabetes são importantes na prevenção primária do DM2 e de suas complicações crônicas. Arq Bras Endocrinol Metab. 2012;56(5):275-84

\section{Descritores}

Pré-diabetes; diabetes melito; complicações microvasculares; doença cardiovascular; prevenção

\section{SUMMARY}

Type 2 diabetes mellitus accounts for $90 \%$ of diabetes cases and is associated with macro- and microvascular complications of high morbidity and mortality. Individuals with increased risk for type 2 diabetes include those with impaired fasting glucose (IFG), impaired glucose tolerance (IGT), and especially those with combined IFG and IGT. These individuals are part of a group known as prediabetes patients. Approximately $25 \%$ of individuals with prediabetes will develop type 2 diabetes in three to five years. Hyperglycemia, in the absence of diabetes, is also associated with increased risk of cardiovascular disease. Studies have shown that changes in lifestyle and drug interventions are effective in delaying or preventing type 2 diabetes in patients with prediabetes. Metformin is the drug of choice when medical treatment is warranted. IGT and IFG are associated with type 2 diabetes and, despite controversy, most studies reinforce the importance of these conditions in the development of micro- and macrovascular disease. Thus, therapeutic interventions in patients with prediabetes are important in primary prevention of type 2 diabetes and its chronic complications. Arq Bras Endocrinol Metab. 2012;56(5):275-84

Keywords

Prediabetes; diabetes mellitus; microvascular complications; cardiovascular disease; prevention
${ }^{1}$ Serviço de Atenção Primária à Saúde, Hospital de Clínicas de Porto Alegre, Universidade Federal do Rio Grande do Sul (UFRGS), Porto Alegre, RS, Brasil ${ }^{2}$ Serviço de Endocrinologia, Hospital de Clínicas de Porto Alegre, UFRGS, Porto Alegre, RS, Brasil
Correspondência para: Cristiane Bauermann Leitão Serviço de Endocrinologia, Hospital de Clínicas de Porto Alegre Rua Ramiro Barcelos, 2350 prédio $12,4^{\circ}$ andar 90035-003 - Porto Alegre, RS, Brasil crisbleitao@yahoo.com.br

Recebido em 23/Abr/2012 Aceito em 19/Jul/2012 


\section{INTRODUÇÃO}

$\mathrm{O}$ diabetes melito (DM) é uma doença crônica associada a complicações micro e macrovasculares de elevada morbimortalidade, requerendo cuidado contínuo, educação permanente e suporte para prevenção de complicações agudas e redução do risco de complicações crônicas (1). O DM do tipo 2 (DM2) é caracterizado por hiperglicemia crônica, resistência insulínica e deficiência relativa na secreção de insulina e é responsável por $90 \%$ dos casos de diabetes (1).

Sob o ponto de vista de saúde pública, o alto custo associado ao cuidado de pessoas com doenças crônicas é uma das questões mais urgentes a serem resolvidas em todo o mundo. As consequências humanas, sociais e econômicas relacionadas ao DM são devastadoras, sendo a doença responsável direta ou indiretamente por aproximadamente 4 milhões de mortes por ano, o que representa $9 \%$ da mortalidade mundial total $(2,3)$. A expectativa de vida é reduzida em média em cinco a sete anos em pacientes com DM2. Os adultos com DM têm risco duas a quatro vezes maior de doença cardiovascular (DCV), doença vascular periférica e acidente vascular cerebral (2). Essas complicações são responsáveis por $65 \%$ da mortalidade por DM e fazem do DM a sétima maior causa de morte nos Estados Unidos. O DM é também a causa mais comum de amputações não traumáticas de membros inferiores, cegueira irreversível e doença renal crônica $(2,3)$.

Embora o risco de desenvolver DM2 ao longo da vida seja alto, nossa capacidade em predizer e prevenir o DM2 na população em geral ainda é limitada. Além disso, a efetividade da detecção precoce por meio de rastreamento em massa de indivíduos assintomáticos ainda não foi definitivamente comprovada (1). Contudo, o DM preenche critérios bem estabelecidos para condições nas quais a detecção precoce é apropriada: é uma doença comum, com prevalência crescente, impõe grande carga aos serviços de saúde, é de fácil diagnóstico, e medidas efetivas para a prevenção de suas complicações podem ser tomadas (1). As metas de prevenção do diabetes são retardar o aparecimento da doença, preservando a função das células beta, e prevenir ou retardar complicações micro e macrovasculares. Os indivíduos comprovadamente com maior risco de desenvolvimento de diabetes incluem aqueles com glicemia de jejum alterada (GJA) e tolerância diminuída à glicose (TDG), fases pré-clínicas da doença, e especialmente aqueles com GJA e TDG combinadas $(1,4)$.

Este artigo tem por objetivo descrever as fases pré-clínicas do DM, revisar sua associação com o desenvolvimento de doença cardiovascular e complicações microvasculares e indicar as intervenções terapêuticas disponíveis para prevenção do DM.

\section{DEFINIÇÃO DE PRÉ-DIABETES, CRITÉRIOS DIAGNÓSTICOS E INDICAÇÕES DE RASTREAMENTO}

Em 1997 e 2003, o The Expert Committee on the Diagnosis and Classification of Diabetes Mellitus reconheceu um grupo intermediário de indivíduos cujos níveis de glicose, embora não satisfaçam os critérios para DM, são elevados para serem considerados normais $(5,6)$. Esse grupo foi definido como GJA e TDG, sendo considerados estágios intermediários na história natural do DM2, hoje conhecido como pré-diabetes. A GJA é diagnosticada pela glicemia após 8 horas de jejum. A TDG somente pode ser identificada por meio da realização do teste oral de tolerância à glicose (TTG) $(1,5,6)$. Conforme padronizado pela Organização Mundial de Saúde (OMS), neste teste é coletada glicemia após jejum de 8 horas e nova coleta é realizada após 2 horas da ingestão de $75 \mathrm{~g}$ de glicose. Durante o teste, o paciente não deve realizar exercício físico ou fumar e, nos três dias anteriores ao teste, a ingestão de carboidratos não deve ser inferior a $150 \mathrm{~g}$, visto que dietas com quantidades menores podem ocasionar resultados falso-negativos (7). Os critérios diagnósticos para DM e a classificação dos distúrbios do metabolismo da glicose estão descritos na tabela 1 .

Tabela 1. Classificação dos distúrbios do metabolismo da glicose

\begin{tabular}{|c|c|c|c|c|}
\hline \multirow[b]{2}{*}{ Categoria } & \multicolumn{4}{|c|}{ Método/Diagnóstico } \\
\hline & $\begin{array}{c}\text { Glicemia de } \\
\text { Jejum }^{*+}\end{array}$ & TTG"+ & $\mathrm{HbA1} \mathrm{c}^{\S \dagger}$ & $\begin{array}{l}\text { Glicemia } \\
\text { casual }^{* *}\end{array}$ \\
\hline Normal & $<100 \mathrm{mg} / \mathrm{dL}$ & $<140 \mathrm{mg} / \mathrm{dL}$ & $<5,7 \%$ & - \\
\hline GJA & $100-125 \mathrm{mg} / \mathrm{dL}$ & $<140 \mathrm{mg} / \mathrm{dL}$ & \multirow{2}{*}{$5,7 \%-6,4 \%$} & - \\
\hline TDG & $<100 \mathrm{mg} / \mathrm{dL}$ & $140-199 \mathrm{mg} / \mathrm{dL}$ & & - \\
\hline DM & $\geq 126 \mathrm{mg} / \mathrm{dL}$ & $\geq 200 \mathrm{mg} / \mathrm{dL}$ & $\geq 6,5 \%$ & $\geq 200 \mathrm{mg} / \mathrm{dL}$ \\
\hline
\end{tabular}

* 0 jejum deve ser de, no mínimo, 8 horas; ${ }^{\dagger} \mathrm{Na}$ ausência de hiperglicemia inequívoca, esses critérios devem ser confirmados pela repetição do teste; ${ }^{\ddagger} 0$ teste deve ser realizado conforme os padrões da Organização Mundial de Saúde, utilizando uma sobrecarga com $75 \mathrm{~g}$ de glicose anidra dissolvida em água; $\$ 0$ teste deve ser feito em laboratório que utiliza um método certificado pelo National Glycohemoglobin Standardization Program e padronizado pelo Diabetes Control and Complications Trial; ** Em pacientes com sintomas clássicos de hiperglicemia ou crise hiperglicêmica.

TTG: teste oral de tolerância à glicose; HDA1c: hemoglobina glicada; GJA: glicemia de jejum alterada; TDG: tolerância diminuída à glicose; DM: diabetes melito. 
Mais recentemente, a Associação Americana de Diabetes (ADA) endossou a utilização da hemoglobina glicada ( $\mathrm{HbAlc}$ ) como método diagnóstico de DM e de pré-diabetes $(1,7)$. Após uma extensa revisão de evidências epidemiológicas, o International Expert Committee (8) definiu os métodos padronizados de medida da HbAlc que podem ser utilizados para sua dosagem. A medida da HbAlc deve ser realizada utilizando-se um método certificado pelo National Glycohemoglobin Standardization Program (NGSP) e padronizado conforme as referências do Diabetes Control and Complications Trial (DCCT). Valores iguais ou acima de 6,5\% são considerados para o diagnóstico de $\operatorname{DM}(1,8,9)$. A HbAlc tem muitas vantagens em relação à glicemia de jejum para o diagnóstico de DM, incluindo maior conveniência (por não ser necessário jejum), maior estabilidade pré-analítica e menor variação diária durante períodos de estresse ou doença. Entretanto, essas vantagens devem ser pesadas diante do alto custo, da menor disponibilidade do teste em determinadas regiões de países em desenvolvimento e da menor correlação entre HbAlc e glicemia média em certos indivíduos. Uma revisão sistemática publicada em 2007 reforça a eficácia da Alc como método diagnóstico do DM, ressaltando as vantagens e desvantagens do seu uso rotineiro (10).

Um estudo do National Health and Nutrition Examination Survey (NHANES) demonstrou que, em uma população de adultos não diabéticos, a glicemia em jejum de $110 \mathrm{mg} / \mathrm{dL}$ corresponde a uma HbAlc de $5,6 \%$, e a de $100 \mathrm{mg} / \mathrm{dL}$ corresponde a uma HbAlc de $5,4 \%$. A análise desses dados demonstrou que um valor de HbAlc de 5,7\%, quando comparado a outros pontos de corte, tem a melhor combinação de sensibilidade (39\%) e especificidade (91\%) para identificar indivíduos com GJA. Assim, pode-se considerar indivíduos com HbAlc entre 5,7\% e 6,4\% como sendo de alto risco para DM futuro e aplicar também a eles o termo pré-diabetes $(1,8,9,11)$. Por outro lado, a baixa sensibilidade do teste é um dos argumentos contra o seu uso na prática clínica (12). Os pontos de corte estabelecidos para HbAlc para detectar indivíduos de alto risco identificarão cerca de $12 \%$ da população adulta dos Estados Unidos; esse número é significativamente menor do que aquele identificado pela glicemia de jejum (25\%) (1). No México, assim como em outros países em desenvolvimento, a ausência de um programa de padronização da HbAlc desencoraja seu uso como método diagnóstico (12). No nosso meio, artigo pu- blicado recentemente demonstrou que o uso isolado da HbAlc para o diagnóstico de DM identifica uma população diferente de pacientes quando comparada com a glicemia de jejum e/ou TTG, podendo não ser suficiente para o diagnóstico correto do DM. Esse artigo sugere que a utilização de algoritmos empregando os testes de glicemia de jejum, TTG e/ou Alc possa ser mais adequada para garantir a correta classificação dos indivíduos com DM (13). García-Alcalá e cols. demonstraram que variações nos resultados da HbAlc referentes à metodologia laboratorial utilizada podem levar a diferenças significativas no manejo de pacientes com DM (14). A menos que os governos e as sociedades médicas trabalhem juntos para construir um programa nacional de padronização da HbAlc, a decisão clínica ainda terá altos níveis de incertezas gerados por problemas metodológicos.

$\mathrm{O}$ rastreamento de DM em adultos assintomáticos deve incluir indivíduos de qualquer idade com índice de massa corporal (IMC) $\geq 25 \mathrm{~kg} / \mathrm{m}^{2}$ e um ou mais fatores de risco para DM, conforme descrito na tabela 2. Tanto a glicemia de jejum como o TTG e a HbAlc podem ser utilizados como método diagnóstico. O TTG tem a vantagem de identificar indivíduos com GJA, TDG, e a combinação de ambos, que têm maior risco de desenvolver a doença. $\mathrm{O}$ intervalo apropriado entre os testes ainda não foi estabelecido. A recomendação de realização do exame a cada três anos baseia-se no fato de que testes falso-negativos seriam repetidos em um tempo relativamente curto, e seria pouco provável que complicações significativas do DM se desenvolvam três anos após um teste negativo (1).

Apesar da importância da identificação do estado de pré-diabetes para determinar indivíduos em risco e dos esforços para determinar o teste diagnóstico mais apropriado, a prevalência de pré-diabetes varia consideravelmente conforme o método utilizado. Estudo recente mostrou que a prevalência dessa condição em indivíduos adultos, > 18 anos, foi de $14,2 \%$ para AlC entre $5,7 \%-6,4 \%, 26,2 \%$ para glicemia de jejum entre $100-125 \mathrm{mg} / \mathrm{dL}, 7,0 \%$ para glicemia de jejum entre $110-125 \mathrm{mg} / \mathrm{dL}$, e de $13,7 \%$ para a glicemia de $2 \mathrm{~h}$ do TTG entre 140-199 mg/dL. A prevalência de pré-diabetes também variou conforme idade, sexo e etnia. Dessa maneira, programas para prevenir o DM devem levar em consideração também questões de equidade, recursos, necessidade e eficiência para atingir seus objetivos (15). 
Tabela 2. Critérios para o rastreamento de DM2 em indivíduos assintomáticos

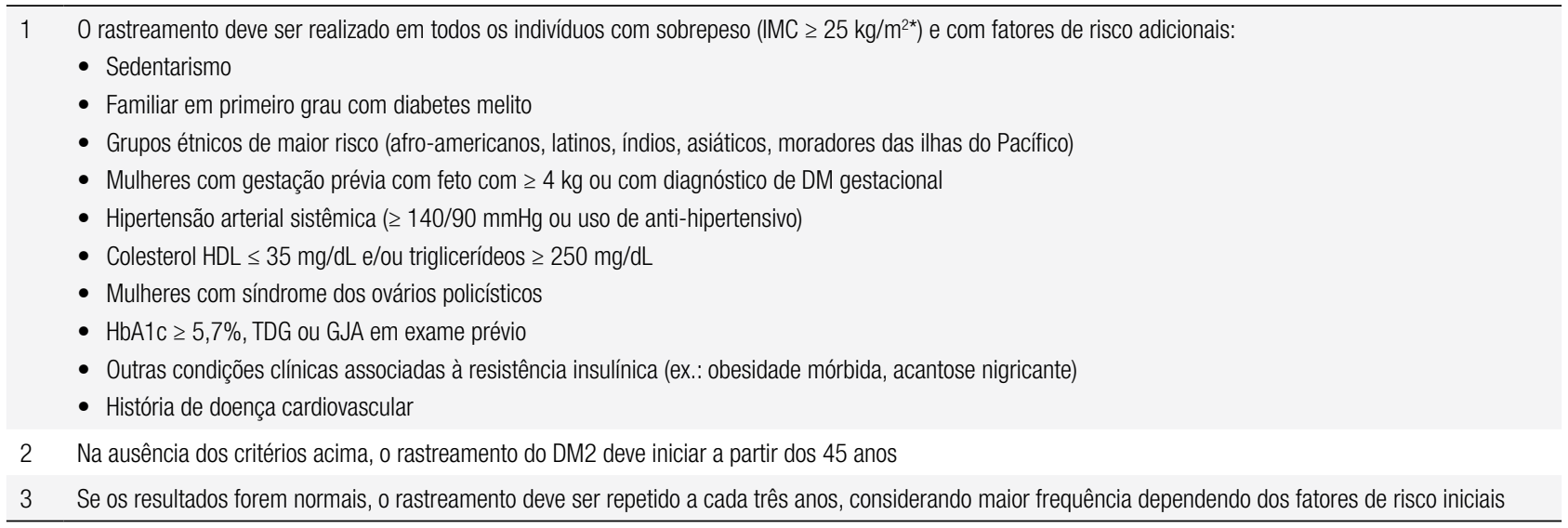

IMC: índice de massa corporal; TDG: tolerância diminuída à glicose; GJA: glicemia de jejum alterada; HDA1c: hemoglobina glicada.

* 0 IMC de risco pode ser menor em alguns grupos étnicos.

\section{PRÉ-DIABETES E RISCO DE DESENVOLVIMENTO DE DIABETES}

Embora a história natural da GJA e da TDG seja variável, aproximadamente $25 \%$ dos indivíduos com qualquer uma dessas alterações desenvolverão DM2 em três a cinco anos (16).

A coorte prospectiva do estudo HOORN demonstrou que a incidência de DM está fortemente relacionada à presença de GJA e TDG na avaliação inicial, particularmente quando essas duas condições estão associadas (4). Gerstein e cols. confirmaram esses achados em uma metanálise recente, em que a GJA e a TDG foram associadas a maior risco de desenvolvimento de DM, tanto em conjunto como isoladamente (17).

Embora a associação da GJA e da TDG com DM esteja bem estabelecida, ainda há controvérsias quanto à associação dessas condições com o desenvolvimento de $\operatorname{DCV}(18)$.

\section{PRÉ-DIABETES E RISCO CARDIOVASCULAR}

O DM é sabidamente um importante fator de risco para mortalidade por DCV (19). A hiperglicemia, na ausência de DM diagnosticado, também foi associada ao aumento no risco de DCV e mortalidade por todas as causas (20). Entretanto, alguns autores sugerem que a glicemia de jejum e a de $2 \mathrm{~h}$ do TTG identificam indivíduos com risco cardiovascular diferente (20).

A impressão de que a glicemia de 2 h do TTG é mais relevante na estratificação do risco cardiovascular dos indivíduos foi decorrente dos resultados do estudo DECODE. Nesse estudo, os pacientes com
DM assintomático ou com TDG determinados pelo TTG apresentaram maior risco de morte por DCV e por todas as causas, independentemente de outros fatores de risco conhecidos e do valor da glicemia de jejum. No entanto, a mortalidade associada à glicemia de jejum foi dependente dos níveis da glicemia após o TTG (20). Esses achados foram confirmados por outros autores $(21,22)$.

Por outro lado, estudos de coorte mais recentes demonstram que o risco de DM2 e de DCV aumenta linearmente com a elevação do valor da glicemia em jejum (23) e que esse risco é ainda maior quando as duas condições (GJA e TDG) estão associadas (24). Wen e cols. demonstraram que a GJA definida pelo critério antigo (110-125 mg/dL) é um fator de risco independente para DCV; associação que não ocorreu com os critérios atuais (100-125 mg/dL) (25). Contrariando os resultados do estudo DECODE, no estudo AusDiab a GJA foi preditora independente para mortalidade cardiovascular, mas a TDG não (26).

Existem ainda evidências de que as duas condições, GJA ou TDG, não estão associadas a maior risco cardiovascular (18), sendo a associação com DCV somente observada naqueles pacientes que evoluíram para DM ao longo do seguimento (27).

Mais recentemente, uma metanálise demonstrou que tanto a GJA (em ambos os pontos de corte: 100 ou $110 \mathrm{mg} / \mathrm{dL}$ ) como a TDG estão associadas a um aumento modesto no risco de DCV. Entretanto, na análise de pacientes com GJA foram incluídos estudos que não realizaram TTG, ou seja, indivíduos com TDG ou até mesmo com DM podem ter sido incluídos nesse 
subgrupo. Nesse estudo, surpreendentemente, as duas condições combinadas (GJA e TDG) não se associaram a um risco cardiovascular maior (28).

Apesar das controvérsias, a maioria dos estudos epidemiológicos reforça a importância da GJA ou da TDG, e poucos sugerem que essas duas condições não confiram maior risco de mortalidade ou de doença cardíaca isquêmica, ou que não acrescentem risco ainda maior após a contabilização de outros fatores de risco cardiovascular associados. Dessa forma, intervenções terapêuticas em pacientes com pré-diabetes podem ser importantes na prevenção primária das complicações crônicas macrovasculares do DM.

\section{PRÉ-DIABETES E COMPLICAÇÕES MICROVASCULARES}

Recentemente, o pré-diabetes vem sendo associado a complicações antes atribuídas somente ao DM. Evidências cumulativas relacionam principalmente a TDG à retinopatia, à nefropatia e à neuropatia (29). Um estudo transversal, que usou a combinação de exame físico, história clínica focada e teste vibratório, encontrou neuropatia em $26 \%$ dos pacientes com DM, em 11,2\% daqueles com TDG e em 3,9\% daqueles com glicemia normal (30).

Muitos estudos documentaram uma prevalência aumentada de microalbuminúria em indivíduos com TDG. A frequência de microalbuminúria é duas a três vezes maior em indivíduos com TDG de diferentes etnias quando comparados a controles com glicemia normal (31). Uma análise do estudo de Framingham demonstrou associação entre glicemia de jejum em níveis subdiabéticos e microalbuminúria, após 24 anos de seguimento, independentemente da pressão arterial sistólica e de outros fatores de risco para microalbuminúria (32). Esses dados sugerem que a hiperglicemia em níveis abaixo daqueles compatíveis com o diagnóstico de DM está associada com anormalidades renais em alguns indivíduos e que essas anormalidades podem preceder o estabelecimento do DM.

O estudo Diabetes Prevention Program (DPP), composto por uma coorte de indivíduos com a associação de GJA e TDG, avaliou a presença de lesões compatíveis com retinopatia diabética (microaneurismas). A retinopatia foi identificada em $12,6 \%$ dos indivíduos que desenvolveram DM após cinco anos de seguimento e em $7,9 \%$ daqueles sem progressão para o DM (33). Nagi e cols. relataram uma prevalência de $12 \%$ de retinopatia diabética não proliferativa em índios Pima com TDG, de 8,3\% naqueles com DM recém-diagnosticado, mas que tinham TTG normal documentado quatro anos antes desse diagnóstico, de 11,2\% naqueles com DM recém-diagnosticado, sendo a prevalência de retinopatia nessa população diretamente relacionada à glicemia de jejum e à glicemia de $2 \mathrm{~h}$ (34). Entretanto, como esses estudos analisaram uma amostra pequena de indivíduos com pré-diabetes e como ainda não se sabe a história natural dessas lesões iniciais, seria precoce demais tentar redefinir os pontos de corte para diabetes com base nesses dados. Coletivamente, esses dados sugerem que estágios iniciais de dano retiniano ocorrem durante o estado de pré-diabetes.

\section{PREVENÇÃO DO DIABETES}

Como o DM2 é fortemente associado a complicações micro e macrovasculares e ao aumento de mortalidade, intervenções que atrasem ou previnam seu estabelecimento podem ser benéficas (29). Três fatores têm sido avaliados na tentativa de prevenir o DM2: exercício físico, perda de peso e terapia medicamentosa. As principais medidas preventivas farmacológicas e não farmacológicas para a prevenção do DM2 são descritas na tabela 3 .

Muitos estudos demonstraram que mudanças no estilo de vida (MEV) são efetivas em retardar ou prevenir o DM2 em pacientes com GJA e TDG. Alguns medicamentos também demonstraram ser efetivos, mas seu benefício não é maior do que a adoção de um estilo de vida mais saudável (1). Entretanto, nem todos os pacientes conseguem atingir as metas de MEV recomendadas e acabam progredindo para o DM. Nesses casos, as intervenções farmacológicas podem ser necessárias. A metformina é a droga mais estudada na prevenção do DM por apresentar baixo custo, fácil disponibilidade $\mathrm{e}$ segurança, e, por ser relativamente bem tolerada, é a droga de escolha nos indivíduos com indicação de tratamento medicamentoso $(1,37,38,41)$.

No entanto, o impacto das intervenções medicamentosas sobre os fatores de risco para $\mathrm{DCV}$ não é tão claro e varia com cada droga individualmente. Além disso, os efeitos sobre os eventos cardiovasculares são pouco conhecidos, e os benefícios e o custo-efetividade a longo prazo do tratamento farmacológico precoce do pré-diabetes versus tratar no momento do diagnóstico de diabetes não são comprovados $(37,38,56)$. 
Tabela 3. Intervenções preventivas para o diabetes melito tipo 2

\begin{tabular}{|c|c|c|c|c|c|}
\hline Estudo & Intervenções & Características da população & $\mathbf{N}$ & $\begin{array}{c}\text { Duração } \\
\text { (anos) }\end{array}$ & $\begin{array}{l}\text { Redução } \\
\text { do risco }\end{array}$ \\
\hline \multicolumn{6}{|l|}{ MEV } \\
\hline Tuomilehto e cols. (35) (FDPS) & Dieta + exercício & TDG e IMC $\geq 25$ & 522 & 3,2 & $58 \%$ \\
\hline FDPS $^{*}(36)$ & Dieta + exercício & $\mathrm{TDG}$ e $\mathrm{IMC} \geq 25$ & 522 & 4 & $36 \%$ \\
\hline $\mathrm{DPP}^{\dagger}(37)$ & Dieta + exercício & GJA e TDG e IMC > 24 & 3.234 & 2,8 & $58 \%$ \\
\hline 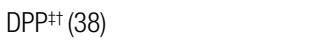 & Dieta + exercício & GJA e TDG e IMC > 24 & 2.766 & 5,7 & $34 \%$ \\
\hline Da Qing (39) & Dieta e/ou exercício & TDG & 577 & 6 & $31 \%-46 \%$ \\
\hline Da Qing ${ }^{\ddagger}(40)$ & Dieta e/ou exercício & TDG & 577 & 20 & $43 \%$ \\
\hline Indian DPP (41) & Dieta + exercício & TDG & 531 & 2,5 & $29 \%$ \\
\hline Toranomon Study (42) & Dieta + exercício & Homens, GJA e TDG & 458 & 4 & $67 \%$ \\
\hline \multicolumn{6}{|l|}{ MEDICAÇÕES } \\
\hline $\operatorname{DPP}^{\dagger}(37)$ & Metformina & GJA e TDG e IMC > 24 & 3.234 & 2,8 & $31 \%$ \\
\hline 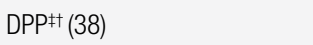 & Metformina & GJA e TDG e IMC > 24 & 2.766 & 5,7 & $18 \%$ \\
\hline Indian DPP (41) & Metformina e/ou exercício & TDG & 531 & 2,5 & $26 \%$ \\
\hline STOP-NIDDM ${ }^{\dagger}(43)$ & Acarbose & TDG, GJA e IMC 25 - 40 & 1.429 & 3,3 & $25 \%$ \\
\hline Voglibose (44) & Voglibose & GJA, TDG e risco para DM & 1.780 & 1 & $40 \%$ \\
\hline XENDOS (45) & Orlistat & IMC $\geq 30$ com ou sem TDG & 3.305 & 4 & $37 \%$ \\
\hline DREAM (46) & Rosiglitazona & GJA ou TDG & 5.269 & 3 & $62 \%$ \\
\hline DREAM (47) & Ramipril & GJA ou TDG & 5.269 & 3 & $* *$ \\
\hline ACT-NOW' $(48)$ & Pioglitazona & TDG, GJA e risco CV & 602 & 2,6 & $81 \%$ \\
\hline NAVIGATOR $^{\dagger}(49)$ & Valsartan & TDG, GJA e DCV ou risco CV & 9.306 & 5 & $14 \%$ \\
\hline NAVIGATOR $^{\dagger}(50)$ & Nateglinida & TDG, GJA e DCV ou risco CV & 9.306 & 5 & $\star \star$ \\
\hline CANOE (51) & Rosiglitazona + metformina & TDG & 207 & 3,9 & $66 \%$ \\
\hline \multicolumn{6}{|l|}{ Estudos com análise post-hoc } \\
\hline HOPE (52) & Ramipril & Indivíduos com DCV & 5.270 & 4,5 & $34 \%$ \\
\hline LIFE (53) & Losartan & Indivíduos com HAS e HVE & 7.998 & 4,7 & $25 \%$ \\
\hline WOSCOPS (54) & Pravastatina & Homens sem DCV & 5.974 & 5 & $30 \%$ \\
\hline HERS (55) & Estrogênio e/ou progesterona & Mulheres pós-menopausa com DCV & 2.029 & 4,1 & $35 \%$ \\
\hline
\end{tabular}

* Extensão do seguimento do Finnish Diabetes Prevention Study (FDPS); † Nas referências 37, 38, 48, 49 e 50 a GJA corresponde à glicemia entre 95-125 mg/dL; na referência 42 a GJA corresponde à glicemia < $140 \mathrm{mg} / \mathrm{dL}$; na referência 43 a GJA corresponde à glicemia entre 100-139 mg/dL; na referência 44 os critérios de inclusão foram glicemia de jejum < 125 mg/dL, glicemia de $2 \mathrm{~h}$ entre 140-199 mg/dL, HbA1c < 6,5\% e pelo menos um fator de risco para diabetes; nas referências 46 e 47 a GJA corresponde à glicemia entre $110-125$ mg/dL; ₹ Estudo após 10 anos de seguimento; ${ }^{\star *}$ Ausência de benefício.

IMC: índice de massa corporal (kg/m²); TDG: tolerância diminuída à glicose; GJA: glicemia de jejum alterada; DM: diabetes melito; CV: cardiovascular; HAS: hipertensão arterial sistêmica; HVE: hipertrofia de ventrículo esquerdo; DCV: doença cardiovascular.

Alguns estudos com intervenções não direcionadas para a glicemia demonstraram um potencial efeito em diminuir o risco de desenvolvimento de diabetes em indivíduos não diabéticos arrolados para avaliar outros desfechos. Nenhum desses estudos foi desenhado para avaliar o desenvolvimento de diabetes e, por esse motivo, ensaios clínicos com o desfecho de diabetes definido a priori devem ser conduzidos para que esses fármacos possam ser recomendados como medidas preventivas $(52-55)$.

A cirurgia bariátrica, com o objetivo de perda de peso, também se mostrou efetiva em reduzir o risco de DM. Um estudo prospectivo mostrou que pacientes submetidos a procedimentos cirúrgicos variados obtiveram redução do risco de DM de $86 \%$ em dois anos, e de $75 \%$ em dez anos, em comparação com o grupo controle (de cuidado padrão) (57). A cirurgia bariátrica também mostrou induzir remissão do DM preexistente. Em um ensaio clínico randomizado, a banda gástrica induziu remissão da doença em $73 \%$ dos pacientes em comparação com $13 \%$ nos pacientes do grupo controle (dieta convencional) (58).

Independentemente da intervenção escolhida, todos os pacientes devem ser acompanhados regularmente para que a adesão às MEV e ao tratamento medicamentoso seja permanente.

O consenso da American Diabetes Association (ADA) recomenda que indivíduos com diagnóstico de TDG, GJA e aqueles com HbAlc entre 5,7\% e 6,4\% sejam referenciados para um programa de suporte vi- 
sando à redução do peso em $5 \%$ a $10 \%$ e ao aumento da frequência de exercício físico para pelo menos 150 minutos/semana de atividade moderada (1). O seguimento e aconselhamento contínuo desses indivíduos parecem ser importantes para o sucesso desse tratamento. Indivíduos com alto risco de desenvolver DM, tais como aqueles com GJA e TDG combinadas, associados a pelo menos um dos fatores de risco: $\mathrm{HbAlc} \geq$ 6\%, hipertensão arterial sistêmica (HAS), HDL baixo, triglicerídeos elevados ou história familiar de DM em parente de primeiro grau, e indivíduos obesos com < 60 anos, podem se beneficiar do uso da metformina (59). O monitoramento do desenvolvimento de DM em indivíduos com pré-diabetes deve ser realizado uma vez por ano (1).

\section{SUMÁRIO DE RECOMENDAÇÕES PARA O MANEJO DE PRÉ-DIABETES}

Os objetivos da intervenção em indivíduos com GJA ou TDG incluem a prevenção de DM2 e de suas complicações, e a redução do risco de DCV $(1,33,59)$. Nesse sentido, as principais recomendações para o manejo de pacientes com pré-diabetes são:

- Indivíduos com risco aumentado para DM2, conforme recomendação da $\mathrm{ADA}$, devem ser submetidos a um dos testes de rastreamento disponíveis. No nosso meio, devido à falta de padronização da $\mathrm{HbAlc}$ e ao maior custo do TTG, o teste recomendado é a glicemia de jejum, e, se esse se apresentar alterado, o TTG.

- O risco cardiovascular deve ser avaliado na anamnese e no exame clínico, e quantificado por meio do Escore de Framingham.

- A medida da microalbuminúria pode ser útil, por ser fator preditor de DCV e influenciar na escolha do agente anti-hipertensivo. A medida anual da microalbuminúria foi recomendada em um consenso do American College of Endocrinology (ACE) e da American Association of Clinical Endocrinologists (AACE) (59). No entanto, não existem evidências de que o tratamento da microalbuminúria nesses indivíduos esteja associado à prevenção de doença renal ou DCV.

- A realização de exame de fundo de olho para rastreamento de retinopatia do DM não é recomendada, visto que, nesses pacientes, somente se identificou a presença de retinopatia leve, sendo pouco provável sua evolução para formas graves antes do desenvolvimento do DM.

- As MEV são a intervenção inicial em indivíduos com pré-diabetes. Os objetivos específicos incluem perda de peso ( $5 \%$ a $10 \%$ do peso corporal), exercício de intensidade moderada ( 150 minutos por semana) e cessação do tabagismo.

- A adesão às MEV deve ser monitorada a cada seis meses, e a avaliação laboratorial com nova glicemia de jejum deve ser realizada anualmente. No caso de glicemia de jejum alterada no exame anual, deve ser solicitado novo TTG ou teste HbAlc.

- Indivíduos que não conseguem obter 5\% a 10\% de perda de peso e/ou normalizar o TTG com MEV unicamente devem receber intervenção medicamentosa.

- A metformina é a droga de primeira escolha, pela sua efetividade e baixo custo, sendo recomendada especialmente em indivíduos com IMC $\geq 35 \mathrm{~kg} / \mathrm{m}^{2}$, idade $<60$ anos, ou que tenham fatores de risco adicionais.

- Os pacientes em uso de metformina devem ser monitorados com a realização de HbAlc semestral.

Um fluxograma de rastreamento e manejo do pré-diabetes, baseado nas recomendações acima, foi elaborado e está descrito na figura 1.

\section{CONSIDERAÇÕES FINAIS}

Esta revisão demonstra a importância clínica e epidemiológica das alterações do metabolismo da glicose como fator de risco para o DM2 e a DCV e reforça a necessidade de que indivíduos de maior risco sejam constante e adequadamente orientados pelos profissionais de saúde a adotarem hábitos de vida saudáveis. Atenção especial deve ser dada àqueles com outros fatores de risco para DCV, como tabagismo, HAS e dislipidemia. Embora o custo-efetividade das estratégias de intervenção ainda deva ser mais estudado, medidas com o objetivo de tratar o pré-diabetes devem ser consideradas, visando à prevenção do DM2 e da DCV.

Agradecimentos: Os autores agradecem aos profissionais de saúde e aos pacientes da Unidade Básica de Saúde Santa Cecília/Hospital de Clínicas de Porto Alegre pelo apoio ao grupo durante as pesquisas desenvolvidas no Serviço, que trouxeram a ideia deste artigo de revisão.

Declaração: os autores declaram não haver conflitos de interesse científico neste estudo. 


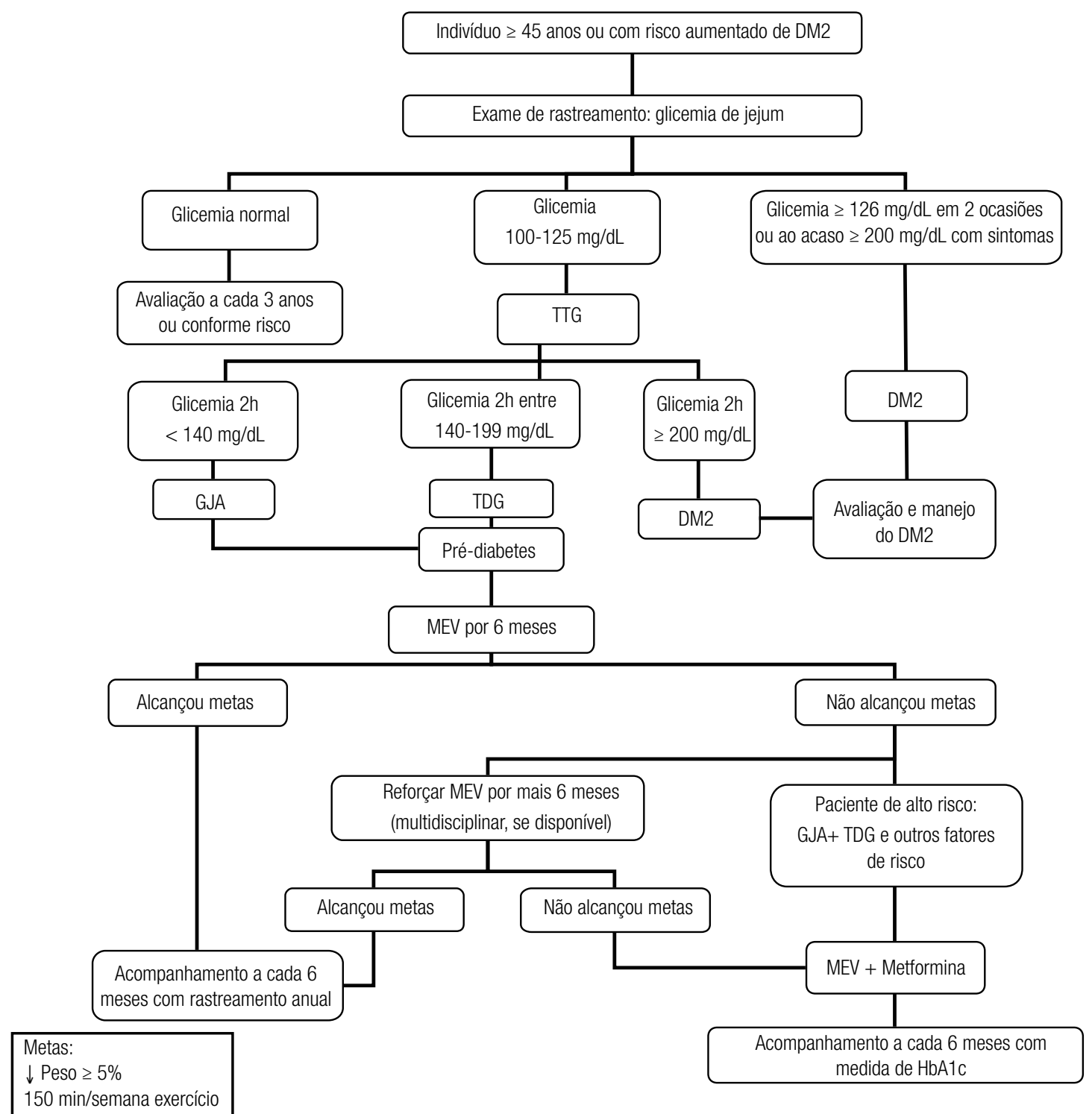

DM2: diabetes melito tipo 2; TTG: teste oral de tolerância à glicose; GJA: glicemia de jejum alterada; TDG: tolerância diminuída à glicose; MEV: mudança no estilo de vida.

Figura 1. Fluxograma para rastreamento e manejo do pré-diabetes.

\section{REFERÊNCIAS}

1. Standards of Medical Care in Diabetes - 2011. Position Statement/American Diabetes Association. Diabetes Care. 2011;33(S1):S11-61.

2. Caderno de Atenção Básica $n^{\circ} 16$ : diabetes mellitus. Ministério da Saúde. Brasília, 2006.

3. Dall T, Mann SE, Zhang Y, Martin J, Chen Y, Hogan P. Economic costs of diabetes in the U.S. in 2007. American Diabetes Association. Diabetes Care. 2008;31:596-615.

4. Vegt F, Dekker JM, Jager A, Hienkens E, Kostense PJ, Stehouwer CDA, et al. Relation of impaired fasting and postload glucose with incident type 2 diabetes in Dutch population: The HOORN Study. JAMA. 2001;285(16):2109-13.

5. Expert Committee on the Diagnosis and Classification of Diabetes Mellitus. Report of the Expert Committee on the Diagnosis and Classification of Diabetes Mellitus. Diabetes Care. 1997;20:1183-97.
6. Genuth S, ALberti KG, Bennett P, Buse J, Defronzo R, Kahn R, et al. Expert Committee on the Diagnosis and Classification of Diabetes Mellitus. Follow up report on the diagnosis of diabetes mellitus. Diabetes Care. 2003;26:3160-7.

7. Gross JL, Silveiro SP, Camargo JL, Reichelt AJ, Azevedo MJ. Diabetes melito: diagnóstico, classificação e avaliação do controle glicêmico. Arq Bras Endocrinol Metab. 2002;46(1):16-26.

8. The International Expert Committee. International Expert Committee report on the role of the A1c assay in the diagnosis of diabetes. Diabetes Care. 2009;32(7):1327-34.

9. American Diabetes Association. Diagnosis and classification of diabetes mellitus. Diabetes Care. 2011;33(S1):S62-9.

10. Bennet CM, Guo M, Dharmage SC. HbA1c as a screening tool for detection of type 2 diabetes: a systematic review. Diabet Med. 2007;24:333-43.

11. Diretrizes da Sociedade Brasileira de Diabetes 2009. Sociedade Brasileira de Diabetes. 3ª edição. São Paulo, 2009. 
12. Gomes-Perez FJ, Aguilar-Salinas CA, Almeda-Valdes P, Cuevas-Ramos D, Garber IL, Rull JA. HbA1c for the diagnosis of diabetes mellitus in a developing country. A position Article. Arch Med Res. 2010;41:302-8.

13. Cavagnolli G, Gross JL, Camargo JL. HbA1c, glicemia de jejum e teste oral de tolerância à glicose no diagnóstico de diabetes: que teste usar? Rev HCPA. 2010;30(4):315-20.

14. García-Alcalá $H$, Ruiz-Argüelles $A$, Cedillo-Carvallo B. Effect of the method to mesure levels of glycated hemoglobin on individual clinical decisions comparison of an immunoassay with high-performance liquid chromatography. Am J Clin Pathol. 2009;132:332-5.

15. James C, Bullard KMK, Rolka DB, Geiss LS, Williams DE, Cowie CC, et al. Implications of Alternative Definitions of Prediabetes for Prevalence in U.S. Adults. Diabetes Care. 2011;34:387-91.

16. Nathan DM, Davidson MB, DeFronzo RA, Heine RJ, Henry RR, Pratley R. Impaired fasting glucose and impaired glucose tolerance: implications for care. Diabetes Care. 2007;30:753-9.

17. Gerstein HC, Santaguida P, Raina P, Morrison KM, Balion C, Hunt $D$, et al. Annual incidence and relative risk of diabetes in people with various categories of dysglycemia: a systematic overview and meta-analysis of prospective studies. Diabetes Res Clin Pract. 2007;78:305-12.

18. Pankow JS, Kwan DK, Duncan BB, Schmidt MI, Couper DJ, Golden $\mathrm{S}$, et al. Cardiometabolic risk in impaired fasting glucose and impaired glucose tolerance: the Atherosclerosis Risk in Communities Study. Diabetes Care. 2007;30:325-31.

19. Diabetes Control and Complications Trial/Epidemiology of Diabetes Interventions and Complications (DCCT/EDIC) Study Research Group. Intensive diabetes treatment and cardiovascular disease in patients with type 1 diabetes. N Engl J Med. 2005;353(25):2643-53.

20. Glucose tolerance and mortality: comparison of WHO and American Diabetes Association criteria - The DECODE study group. Lancet. 1999;354:617-21.

21. Bartnik M, Malmberg K, Norhammar A, Tenerz A, Ohrvik J, Ryden L. Newly detected abnormal glucose tolerance: an important predictor of long-term outcome after myocardial infarction. Eur Heart J. 2004;25:1990-7.

22. Tominaga $M$, Eguchi $H$, Manaka $H$, Igarashi $K$. Impaired glucose tolerance is a risk factor for cardiovascular disease, but not impaired fasting glucose: the Funagata Diabetes Study. Diabetes Care. 1999;22:920-4.

23. Shaw JE, Zimmet PZ, Hodge AM, Courten M, Dowse GK, Chitson $P$, et al. Impaired fasting glucose: how low should it go? Diabetes Care. 2000;23:34-9.

24. Lim SC, Tai ES, Tan BY, Chew SK, Tan CE. Cardiovascular risk profile in individuals with borderline glicemia. Diabetes Care. 2000;23:278-82.

25. Wen CP, Cheng TYD, Tsai SP, Hsu HL, Wang SL. Increased mortality risks of pre-diabetes (impaired fasting glucose) in Taiwan. Diabetes Care. 2005;28:2756-61.

26. Barr ELM, Zimmet PZ, Welborn TA, Jolley D, Magliano DJ, Dunstan DW, et al. Risk of cardiovascular and all-cause mortality in individuals with diabetes mellitus, impaired fasting glucose, and impaired glucose tolerance: the Australian Diabetes, Obesity, an Lifestyle Study (AusDiab). Circulation. 2007;116:151-7.

27. Rijkelijkhuizen JM, Nijpels G, Heine RJ, Bouter LM, Stehouwer CDA, Dekker JM. High risk of cardiovascular mortality in individuals with impaired fasting glucose is explained by conversion to diabetes: the Hoorn Study. Diabetes Care. 2007;30:332-6.

28. Ford ES, Zaho G, Li C. Prediabetes and the risk for cardiovascular disease. A systematic review of the evidence. JACC. 2010;55:1310-7.

29. Singleton JR, Smith AG, Russel JW, Feldman EL. Microvascular complications of impaired glucose tolerance. Diabetes. 2003;52:2867-73.
30. Franklin GM, Kahn LB, Bender J, Marshall JA, Hamman F. Sensory neuropathy in non-insulin-dependent diabetes mellitus. The San Luis Valley Diabetes Study. Am J Epidemiol. 1990;131:633-43.

31. Nelson RG, Kunzelman CL, Pettitt DJ, Saad MF, Bennett PH, Knowler WC. Albuminuria in Type 2 (non-insulin-dependent) diabetes mellitus and impaired glucose tolerance in Pima indians. Diabetologia. 1989;32:870-86.

32. Meigs JB, Dágostinho RB, Nathan DM, Rifai N, Wilson PW. Longitudinal Association of glycemia and microalbuminuria: the Framingham Offspring Study. Diabetes Care. 2002;25:977-83.

33. The prevalence of retinopathy in impaired glucose tolerance and recent-onset diabetes in the Diabetes Prevention Program. Diabetes Prevention Program Research Group. Diabetic Medicine. 2007;24:137-44.

34. Nagi DK, Pettitt DJ, Bennett PH, Klein R, Knowler WC. Diabetic retinopathy assessed by fundus photography in Pima indians with impaired glucose tolerance and NIIDM. Diabetic Medicine. 1997;14(6):449-56.

35. Tuomilehto J, Lindström J, Eriksson JG, Valle TT, Hämäläinen H, Ilanne-Parikka $\mathrm{P}$, et al. Prevention of type 2 diabetes by changes in lifestyle among subjects with impaired glucose tolerance. The Finish Diabetes Prevention Study. N Engl J Med. 2002;344:1343-50.

36. Lindström J, llanne-Parikka P, Peltonen M, Aunola S, Eriksson JG, Hemiö K, et al. Sustained reduction in the incidence of type 2 diabetes by lifestyle intervention: follow up of the Finnish Diabetes Prevention Study. Lancet. 2006;368:1673-9.

37. DPP Research Group: reduction in the incidence of type 2 diabetes with lifestyle intervention or metformin. $\mathrm{N}$ Engl J Med. 2002;346:393-403.

38. DPP Research Group: 10-year follow-up of diabetes incidence and weight loss in the Diabetes Prevention Program Outcomes Study. Lancet. 2009;374:1677-86.

39. Pan XR, Li GW, Hu YH, Wang JX, Yang WY, An ZX, et al. Effects of diet and exercise in preventing NIDDM in people with impaired glucose tolerance: the Da Qing IGT and Diabetes Study. Diabetes Care. 1997;20:537-44.

40. Li G, Zhang P, Wang J, Gregg EW, Yang W, Gong Q, et al. The long-term effect of lifestyle interventions to prevent diabetes in the China Da Oing Diabetes Prevention Study: a 20-year follow-up study. Lancet. 2008;371:1783-9.

41. Ramachandran A, Snehalatha C, Mary S, Mukesh B, Bhaskar AD, Vijay V. The Indian Diabetes Prevention Programme shows that lifestyle modification and metformin prevent type 2 diabetes in Asian Indian subjects with impaired glucose tolerance (IDDP-1). Diabetologia. 2006;49:289-97.

42. Kosaka K, Noda M, Kuzuya T. Prevention of type 2 diabetes by lifestyle intervention: a Japanese trial in IGT males. Diabetes Res Clin Pract. 2005;67:152-62.

43. Chiasson JL, Josse RG, Gomis R, Hanefeld M, Karasik A, Laakso M. STOP-NIDDM Trial Research Group: Acarbose for prevention of type 2 diabetes mellitus: the STOP-NIDDM randomized trial. Lancet. 2002;359:2072-7.

44. Kawamori R, Tajima N, Iwamoto Y, Kashiwagi A, Shimamoto K, Kaku K. Voglibose Ph-3 Study Group: Voglibose for prevention of type 2 diabetes mellitus: a randomised, double-blind trial in Japanese individuals with impaired glucose tolerance. Lancet. 2009;373:1607-14.

45. Torgerson JS, Hauptman J, Boldrin MN, Sjöström L. XENical in the prevention of diabetes in obese subjects (XENDOS) study: a randomized study of orlistat as an adjunct to lifestyle changes for the prevention of type 2 diabetes in obese patients. Diabetes Care. 2004;27:155-61.

46. DREAM (Diabetes REduction Assessment with ramipril and rosiglitazone Medication) Trial Investigators, Gerstein HC, Yusuf S, 
Bosch J, Pogue J, Sheridan P, Dinccag N, et al. Effect of rosiglitazone on the frequency of diabetes in patients with impaired glucose tolerance or impaired fasting glucose: a randomized controlled trial. Lancet. 2006;368:1096-105.

47. Gerstein HC, Yusuf S, Bosch J, Pogue J, Sheridan P, Dinccag N, et al. DREAM (Diabetes REduction Assessment with ramipril and rosiglitazone Medication) Trial Investigators: effect of ramipril on the incidence of diabetes. N Eng J Med. 2006;355:1551-62.

48. DeFronzo RA, for ACT NOW Study Group. ACTos NOW Study for the Prevention of Diabetes (ACT NOW) Study. Late-breaking abstract presented at 68th Annual Scientific Sessions of the American Diabetes Association, 6 June 2008, San Francisco, CA.

49. The NAVIGATOR Study Group: effect of valsartan on the incidence of diabetes and cardiovascular events. $\mathrm{N}$ Eng $\mathrm{J}$ Med. 2010;362:1477-90.

50. The NAVIGATOR Study Group: effect of nateglinide on the incidence of diabetes and cardiovascular events. $\mathrm{N}$ Eng $\mathrm{J}$ Med. 2010;362:1477-90.

51. Zinman B, Harris SB, Neuman J, Gerstein HC, Retnakaran RR, Raboud J, et al. Low-dose combination therapy with rosiglitazone and metformin to prevent type 2 diabetes mellitus (CANOE trial): a double-blind randomized controlled study. Lancet. 2010;376:103-11.

52. Yusuf S, Gerstein H, Hoogwerf B, Pogue J, Bosch J, Wolffenbuttel $\mathrm{BH}$, et al. HOPE Study Investigators: Ramipril and the development of diabetes. JAMA. 2001;286:1882-5.
53. Lindholm LH, Ibsen $H$, Borch-Johnsen K, Olsen MH, Wachtell K, Dahlof $B$, et al.; for the LIFE study group: risk of new-onset diabetes in the Losartan Intervention For Endpoint Reduction in Hypertension study. J Hypertens. 2002;20:1879-86.

54. Freeman DJ, Norrie J, Sattar N, Neely RD, Cobbe SM, Ford I, et al. Pravastatin and the development of diabetes mellitus: evidence for a protective treatment effect in the West of Scotland Coronary Prevention Study. Circulation. 2001;103:357-62.

55. Kanaya AM, Herrington D, Vittinghoff E, Lin F, Grady D, Bittner V, et al. Heart and Estrogen/progestin Replacement Study: glycemic effects of postmenopausal hormone therapy: the Heart and Estrogen/Progestin Replacement Study: a randomized, double-blind, placebo-controlled trial. Ann Intern Med. 2003;138:1-9.

56. Chiasson JL, Josse RG, Gomis R, Hanefeld M, Karasik A, Laakso M. STOP-NIDDM Trial Research Group: Acarbose treatment and the risk of cardiovascular disease and hypertension in patients with impaired glucose tolerance. The STOP-NIDDM Trial. JAMA. 2003;290:486-94.

57. Sjostrom L. Lifestyle, diabetes, and cardiovascular risk factors 10 years after bariatric surgery. N Engl J Med. 2004;351:2683-93.

58. Dixon JB, O'Brien PE, Playfair J, Chapman L, Schachter LM, Skinner $S$, et al. Adjustable gastric banding and conventional therapy for type 2 diabetes: a randomized controlled trial. JAMA. 2008;299:316-23.

59. Garber AJ, Handelsman Y, Einhorn D, Bergman DA, Blommgarden ZT, Fonseca V, et al. Prediabetes Consensus Statement. Endocr Pract. 2008;14:933-45. 